\title{
Dilemmatic presentation of linear IgA disease: a case report
}

\author{
Roopashri Rajesh Kashyap', Gopakumar R. Nair², Rajesh Shanker Kashyap³ \\ ${ }^{I}$ Department of Oral Medicine and Radiology, A. J. Institute of Dental Sciences, Mangalore 575004, India. \\ ${ }^{2}$ Department of Oral Medicine and Radiology, K. D. Dental College \& Hospital, Mathura 281006, India. \\ ${ }^{3}$ Department of Periodontics, Yenepoya Dental College, Mangalore 575018, India.
}

Correspondence to: Dr. Roopashri Rajesh Kashyap, Department of Oral Medicine and Radiology, A. J. Institute of Dental Sciences, Mangalore 575004, India. E-mail: roopashri.r.k@gmail.com

How to cite this article: Kashyap RR, Nair GR, Kashyap RS. Dilemmatic presentation of linear IgA disease: a case report. Stomatological Dis Sci 2017;1:93-6.

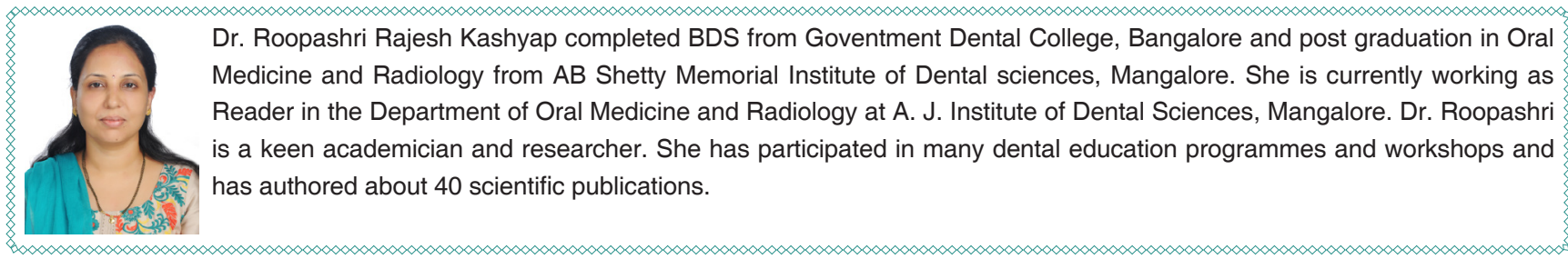

\section{Article history:}

Received: 13 May 2016

Accepted: 7 Dec 2016

Published: 29 Sep 2017

\section{Key words:}

Linear IgA disease,

symblepharon,

immunofluorescence,

dapsone

\begin{abstract}
Linear IgA disease is a rare blistering disease affecting the skin and less often the oral mucosa. This disease has a varied clinical presentation especially when the disease affects the oral mucosa. Certain drugs, autoimmune diseases, infection, chronic renal insufficiency, and malignancies have been implicated as the etiologic factors of this disease. The gold standard for diagnosis of linear IgA disease is direct immunofluorescence on fresh tissue. The immunofluorescence will demonstrate a homogeneous linear deposition of immunoglobulin A along the basement membrane. Multiple therapeutic agents, including corticosteroids, dapsone, sulfapyridine, and colchicine (either alone or in combination), have been used. The authors report a case of linear IgA disease with ambiguous features of pemphigus and cicatricial pemphigoid.
\end{abstract}

\section{INTRODUCTION}

Linear $\lg A$ disease $(L A D)$ is a rare chronic autoimmune subepithelial mucocutaneous bullous disorder characterized by linear deposition of $\lg A$ immunoreactants at the cutaneous or mucosal basement membrane zone $(B M Z) \cdot{ }^{[1]}$ LAD is one of the rarer blistering diseases, with an incidence of only 0.5 per one million in Western Europe. LAD is more commonly found in other parts of the world, including

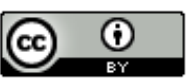

This is an open access article licensed under the terms of Creative Commons Attribution 4.0 International License (https://creativecommons.org/licenses/by/4.0/), which permits unrestricted use, distribution, and reproduction in any medium, as long as the original author is credited and the new creations are licensed under the identical terms.

For reprints contact: service@oaepublish.com

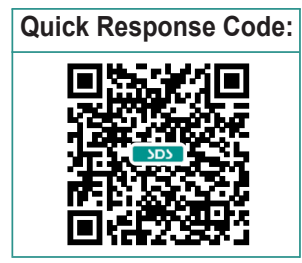


China, Southeast Asia, and Africa. ${ }^{[2]}$ Unfortunately, accurate data regarding LAD is not available.

LAD can manifest at any age, although there are two peaks of onset, one in early childhood and the other after the age of 60 years. ${ }^{[2]}$ LAD is more common among females than males. ${ }^{[3]}$ The clinical presentation consists of plaques, papules, vesicles, and bullae and can develop on the skin of the trunk, shoulders, groin, and lower extremities. ${ }^{[1]}$ Involvement of mucous membrane of eye and mouth are documented in approximately $80 \%$ of cases. Some patients present with a clinical phenotype similar to that of cicatricial pemphigoid. ${ }^{[4]}$ The causes of LAD are not clear. Certain drugs, autoimmune diseases, infection, chronic renal insufficiency, and malignancies have all been reported to be associated with LAD..$^{[5]}$ Here we report a case of $L A D$ with ambiguous features of pemphigus and of cicatricial pemphigoid.

\section{CASE REPORT}

A 60-year-old female patient presented to our clinic with the chief complaint of painful ulcers on her lips of 3 months duration. The patient had initially noticed small fluid-filled blisters measuring less than $1 \mathrm{~cm}$ in diameter. These vesicles ruptured within $24 \mathrm{~h}$ and had formed ulcers. These ulcers were associated with an occasional burning sensation. Similar vesicles followed by ulcerations were formed in different parts of the buccal mucosa and lips. These ulcers healed within 2 days. The ulcers were not associated with any episodes of fever either before or after the onset of the lesions. The lesion on the lip was noted 3 months before her presentation. The lip lesion gradually progressed to attain the size demonstrated at her presentation. The lesion was associated with edema and a burning sensation of the lips, and she had difficulty with food intake. The patient also gave a history of blisters in the vagina, scalp, face, and extremities one year prior, which were healed by medications prescribed by her dermatologist. Details of medication prescribed were not available. The patient also complained of eye lesions and skin lesions for 3 months.

Examination revealed sunken eyes with areas of erythema present over the lateral part of the sclera of the left eye. Trichiasis and symblepharon of the lower palpebrae were present [Figure 1]. Continuous lacrimation of both eyes was present. Few scars of healing lesions were found on the skin of the hands and legs. A submandibular lymph node was palpable on the left side. This submandibular node was solitary, firm, non-tender, and mobile.

Both of the lips were erythematous and swollen, which

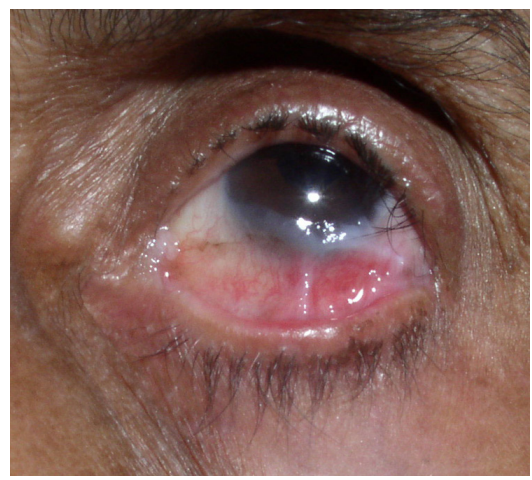

Figure 1: Symblepharon of lower eyelid

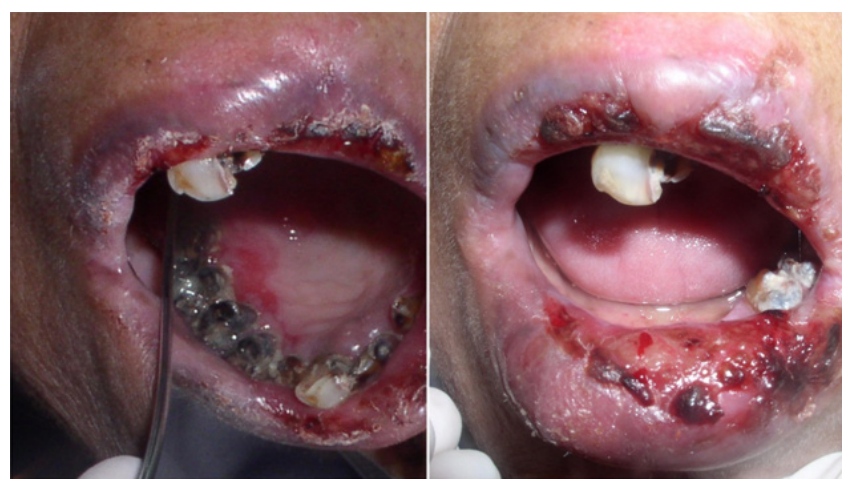

Figure 2: Erosive lesions on palate and ulcerative lesions on lips with encrustations

was more predominant on the lower lip. The lower lip was everted. A large ulcerated area, with surface encrustations, was noted over the lower lip. Mouth opening was painful. On intraoral examination, a large superficial irregular ulceration was observed on the lower lip measuring about three $\mathrm{cm}$ in diameter. The base of the ulcer was covered with encrustations [Figure 2].

On palpation, the ulcer was superficial, severely tender, and bled when touched. There were 2-3 similar ulcers noted on the upper lip with areas of encrustations. Erosive lesions and ulcers, covered by a necrotic slough, were also present on the palate. Generalized erythema was noted predominantly on the buccal mucosa. The tongue was depapillated. The gingiva was erythematous, soft, enlarged, and bled on probing. The differential diagnosis included cicatricial pemphigoid or pemphigus. Pemphigus was considered because the patient's history had revealed vesicles which ruptured within $24 \mathrm{~h}$ of onset. The patient was prescribed Omnacortil ${ }^{\circledR}$ tablets, anticandidal mouth paint, and chlorhexidine mouthwash for oral lesions. Lacrisol Eye Drops $^{\circledR}$ were prescribed for the eye lesions. Iron and folic acid supplements were also given.

A peripheral smear taken from the perilesional area consisted mainly of acute inflammatory cells. A few 


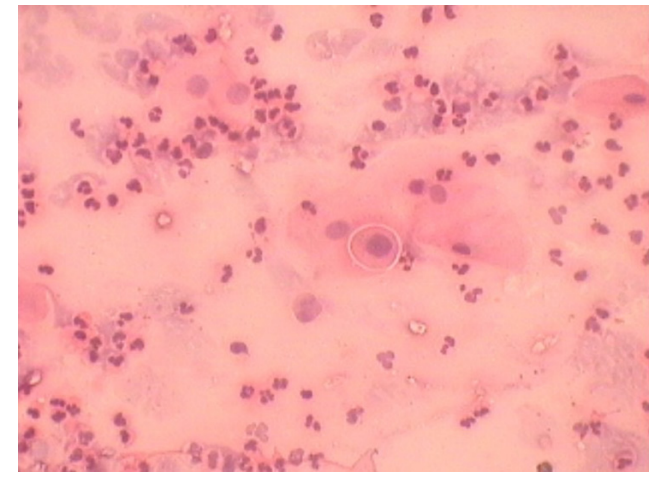

Figure 3: Cytological smear showing Tzanck cells $(\mathrm{HE}, \times 40)$

epithelial cells were also seen, some of which appeared round with a dense hyperchromatic nucleus suggestive of acantholytic Tzanck cells [Figure 3]. Cytologic features were suggestive of pemphigus vulgaris. The incisional biopsy revealed connective tissue devoid of epithelium. Connective tissue was made up of loosely arranged fibrous tissue showing infiltration of chronic inflammatory cells. As the patient did not respond to systemic corticosteroid therapy, further investigation of immunofluorescence was suggested. Biopsy samples were taken from the mucosal part of the lip, as well as from the arm.

Direct immunofluorescence staining of the lip and an arm specimen showed moderately strong linear deposits of $\lg A$ at the $B M Z$ in the floor pattern at a split site, but no evidence of $\lg G$, IgM, C3, or fibrinogen. Indirect immunofluorescence was negative for $\lg \mathrm{g}$ at 1:10 and 1:80 concentration. The final diagnosis was given as LAD. The patient was treated with dapsone $100 \mathrm{mg}$. Simultaneously the patient was also treated with a maintenance dose of Omnacortil ${ }^{\circledR}$ tablets, anticandidal mouth paint/chlorhexidine mouthwash for the oral lesions, and Lacrisol Eye Drops ${ }^{\circledR}$ for the eyes. Partial remission of lesions was observed within 4 weeks, and after 6 months the lesions on the lips and oral mucosa had healed with scarring. The patient was advised to have her remaining teeth extracted, followed by prosthodontic rehabilitation with complete dentures.

\section{DISCUSSION}

LAD is a rare and chronic autoimmune subepithelial mucocutaneous bullous disorder that is diagnosed by the demonstration of a homogeneous linear band of $\operatorname{IgA}$ at the BMZ. This condition was originally thought to be a variant of bullous pemphigoid orherpetiform dermatitis. However, during the 1970s, LAD was recognized as a separate, distinct entity. LAD shows a peak incidence between 60-65 years of age, with a 2:1 predilection for females. ${ }^{[6]}$
The most important antigen in LAD is the antigen BP180. The IgA autoantibodies from LAD patients are directed against thehemidesmosomal transmembrane glycoprotein BP180. The "gold standard" for diagnosis of LAD is direct immunofluorescence on fresh tissue, which shows a homogeneous linear deposition of IgA along the basement membrane. ${ }^{[6]}$

The clinical presentation of LAD is usually heterogeneous. To arrive at a diagnosis from the clinical signs and symptoms is difficult. LAD predominantly involves the skin, although oral manifestations have occasionally been reported. Almost two-thirds of the patients have oral lesions, which affects the hard and soft palate, tonsillar pillars, buccal mucosa, tongue, and gingiva. ${ }^{[6]}$ Our patient presented with predominant oral lesions and minimal skin lesions. It should be noted that because the main clinical features of both cicatricial pemphigoid and mucosal LAD are similar and as there is considerable overlap in the target antigens, it has been proposed that these diseases can be grouped together. The mucosal form of LAD should be considered a variant of cicatricial pemphigoid. ${ }^{[7]}$ As per thecriteria given by Egan and Zone ${ }^{[8]}$ LAD may be diagnosed based on: (1) the presence of a vesicular or bullous eruption, usually confined to the skin, but which may involve the mucous membranes; (2) the presence of a subepidermal vesicle with a predominantly neutrophilic infiltrate on histology of lesional skin; and (3) the presence of BMZ specific IgA antibody deposited in a linear pattern in the absence of other immunoglobulins on DIF of perilesional skin. ${ }^{[8]}$

As mentioned by Betts et al.,, ${ }^{[7]}$ a diagnosis of LAD should be made when there is linear IgA deposition at the basement membrane zone as the sole immunoreactant present. LAD frequently fails to respond to topical or systemic corticosteroids alone. Failure of oral vesiculobullous disease to respond to corticosteroid therapy has been cited as being suggestive of the diagnosis. In our patient, IgA was the sole reactant present at $B M Z$ and also the patient did not respond to corticosteroid therapy ruling out the possibility of cicatricial pemphigoid..$^{[7]}$ Also, scarring is a common feature of cicatricial pemphigoid; few reports mention that scarring can also be a manifestation of LAD. $^{[7-9]}$ Scarring similar to cicatricial pemphigoid but Tzanck cells similar to pemphigus was the confusing feature of this case.

LAD is considered to be a difficult disease to treat, though many different approaches to treatment have been attempted. Multiple therapeutic agents, including dapsone, sulfapyridine, and colchicine, either alone or in combination, have been used. ${ }^{[3]}$ Dapsone is the 
typical first-line systemic treatment for LAD. Dapsone is an antileprosy/anti-malarial drug and is often used because of its bacteriostatic, anti-inflammatory, and immunomodulating properties. ${ }^{[1]}$ Our patient responded to dapsone therapy. Complete remission of the lesions occurred, but with some amount of scarring.

In conclusion, LAD is a chronic disease with a varied clinical presentation which may be a challenge for diagnosticians. Our patient presented with features of pemphigus and cicatricial pemphigoid and was finally diagnosed as LAD. Immunofluorescence plays a critical role in the diagnosis of this condition. Immunofluorescence studies should be made a mandatory part of the diagnostic workup in the evaluation of chronic vesiculobullous diseases.

\section{Acknowledgments}

We thank Dr. Jon Wagner for his contribution in language editing.

\section{Financial support and sponsorship}

None.

\section{Conflicts of interest}

There are no conflicts of interest.

\section{Patient consent}

Obtained.

\section{Ethics approval}

Procedures conducted were in accordance with the institutional ethical committee and after obtaining the patient's consent.

\section{REFERENCES}

1. Angiero F, Benedicenti S, Crippa R, Magistro S, Farronato D, Stefani M. A rare case of desquamative gingivitis due to linear IgA disease: morphological and immunofluorescence features. In Vivo 2007;21:1093-8.

2. Venning VA. Linear IgA disease: clinical presentation, diagnosis, and pathogenesis. Immunol Allergy Clin North Am 2012;32:245-53.

3. Cohen DM, Bhattacharyya I, Zunt SL, Tomich CE. Linear IgA disease histopathologically and clinically masquerading as lichen planus. Oral Surg Oral Med Oral Pathol Oral Radiol Endod 1999;88:196-201.

4. Weinberg MA, Insler MS, Campen RB. Mucocutaneous features of autoimmune blistering diseases. Oral Surg Oral Med Oral Pathol Oral Radiol Endod 1997;84:517-34.

5. Thai IC, Chu CY, Chen HJ, Wang LF, Chiu HC. Linear IgA bullous dermatosis: a clinical study of 16 cases at National Taiwan University Hospital. Dermatol Sin 2010;28:21-6.

6. Lewis MA, Yaqoob NA, Emanuel C, Potts AJ. Successful treatment of oral linear IgA disease using mycophenolate. Oral Surg Oral Med Oral Pathol Oral Radiol Endod 2007;103:483-6.

7. Betts A, Yeoman CM, Farthing PM. Oral mucosal involvement as the sole or main manifestation of linear IgA disease: case report and review of the literature. Oral Surg 2009;2:198-204.

8. Egan CA, Zone JJ. Linear IgA bullous dermatosis. Int $J$ Dermatol 1999;38:818-27.

9. Ramos-Castellón C, Ortiz-Nieva G, Fresán F, Villalvazo L, Garfias Y, Navas A, Jiménez-Martínez MC. Ocular involvement and blindness secondary to linear IgA dermatosis. J Ophthalmol 2010;2010:85-92. 